\title{
5 Schriftliche Befragung von Arztpraxen, Kliniken, Pflegeheimen und Pflegediensten
}

Aufbauend auf den Ergebnissen der Fokusgruppendiskussion wurde eine schriftliche Befragung mit Hausärzten, Kliniken, Pflegeheimen und Pflegediensten in der Region Oberfranken durchgeführt. Hierzu wurden aus den Erkenntnissen der Fokusgruppen berufsgruppenspezifische Fragebögen erstellt, um damit ein repräsentativeres Meinungsbild der einzelnen Leistungserbringergruppen abbilden zu können.

\subsection{Methodik}

Im Zeitraum von Juli bis September 2015 wurde eine postalische Befragung mit Vertretern aus Arztpraxen, Kliniken, Pflegeheimen und Pflegediensten durchgeführt. Die Fragebögen wurden speziell für diese Studie entwickelt. Entsprechend der vier Berufsgruppen gab es vier individuelle Fragebögen, die jeweils für die Berufsgruppe relevante Fragen beinhalteten. Durch die schriftliche Befragung konnte jeglichem Einfluss der Antworten durch die Anwesenheit eines Interviewers vorgebeugt werden. Um trotzdem weitestgehend eine Verständlichkeit der Fragen zu gewährleisten, wurden die Fragebögen auf Grundlage der Erkenntnisse aus den Fokusgruppen skizziert, diskutiert und umfassend mit Vertretern aller Berufsgruppen auf ihre Verständlichkeit und Sinnhaftigkeit geprüft. Ein erheblicher Teil der Studienzeit wurde in die Anpassung und Optimierung der Fragebögen investiert. Diese wur- 
den schwerpunktmäßig an Leistungserbringer in der Metropolregion Erlangen-Nürnberg-Fürth verschickt. In anderen Regionen Oberfrankens u.a. in der Region Bayreuth wurde zusätzlich ein Teil der Fragebögen verteilt. Die potentiellen Teilnehmer wurden schriftlich und persönlich kontaktiert und informiert. Ebenfalls wurde mit mehreren postalischen und persönlichen Remindern zur Teilnahme motiviert. Die Fragebögen enthielten Fragen zur aktuellen Versorgungssituation von Homecare-Patienten, zur Zusammenarbeit der Berufsgruppen untereinander, und zu den Potentialen und Risiken der Zusammenarbeit mit externen Homecare-Dienstleistern.

\subsection{Studienpopulation}

An der Befragung beteiligten sich 210 niedergelassene Ärzte, 6o Mitarbeiter aus Kliniken sowie 69 bzw. 33 Mitarbeiter aus Pflegeheimen und -diensten. Insgesamt nahmen somit 372 Personen teil.

Aus Tabelle 6 wird ersichtlich, dass die vertretenen Institutionen regelmäßig Patienten aus allen Homecare-Therapiebereichen betreuen. Auf der Basis von Schätzungen der Befragten indizieren die Prozentzahlen, welcher Anteil der Befragten mindestens einen Patient in dem jeweiligen Therapiebereich betreut (bzw. dessen Institution). Dies lässt einen Aufschluss über die ungefähren Mengenverteilungen zu, wobei anzumerken ist, dass bei der Befragung weiter nach der geschätzten Patientenzahl differenziert wurde.

Jeweils am häufigsten versorgt werden Wundpatienten. Die geringsten Patientenzahlen finden sich im Bereich Tracheostoma. Alle Berufsgruppen betreuen zumeist zwischen ein bis zehn Patienten pro Therapiebereich (pro

Tab. 6 Teilnehmerzahlen nach Berufsgruppe und Anteile nach Homecare-Bereich und
Versorgungsgebiet

\begin{tabular}{|lcccc}
\hline Teilnehmer aus & Arztpraxis & Klinik & Pflegeheim & Pflegedienst \\
\hline Teilnehmerzahl & 210 & 60 & 69 & 33 \\
\hline Homecare-Bereiche & & & & \\
Wundversorgung & $85 \%$ & $62 \%$ & $77 \%$ & $94 \%$ \\
Inkontinenz & $80 \%$ & $42 \%$ & $66 \%$ & $91 \%$ \\
Uro-/Enterostoma & $77 \%$ & $36 \%$ & $46 \%$ & $75 \%$ \\
Enterale Ernährung & $67 \%$ & $53 \%$ & $72 \%$ & $68 \%$ \\
Parenterale Ernährung & $50 \%$ & $59 \%$ & $45 \%$ & $38 \%$ \\
Tracheostoma & $36 \%$ & $33 \%$ & $33 \%$ & $37 \%$ \\
\hline Versorgungsgebiet & & & & \\
Großstädtisch & $41 \%$ & $77 \%$ & $27 \%$ & $23 \%$ \\
Kleinstädtisch & $30 \%$ & $17 \%$ & $37 \%$ & $43 \%$ \\
Ländlich & $28 \%$ & $6 \%$ & $36 \%$ & $33 \%$ \\
\hline
\end{tabular}


Quartal). In der stationären Pflege werden schwerpunktmäßig Pflegebedürftige im Bereich der Wund- und Inkontinenzversorgung betreut. Die ambulanten Pflegedienste betreuen relativ gesehen die meisten Homecare-Patienten. Auch hier liegt der Schwerpunkt im Bereich Wund- und Inkontinenzversorgung. Die ambulante Pflege nimmt zudem eine deutliche Leistungserhöhung im medizinisch behandlungspflegerischen Bereich wahr (55\%). Etwa 36\% der Befragten aus der ambulanten Pflege schätzen diese Erhöhung als mäßig ein, für 9\% ist kein Unterschied feststellbar.

Teilnehmer aus Arztpraxen und Kliniken kamen überwiegend aus großstädtischen Versorgungsgebieten mit mehr als 50.00o Einwohnern. Pflegeheime und -dienste waren mehrheitlich aus kleinstädtischen Regionen mit 10.000 bis 50.000 Einwohnern vertreten, wobei ein nahezu gleichgroßer Anteil an Teilnehmern aus ländlichen Gegenden mit weniger als 10.000 Einwohnern gewonnen werden konnte.

\subsubsection{Teilnehmende niedergelassene Ärzte}

Die Mehrheit der niedergelassenen Ärzte (66\%) war zwischen 40 und 59 Jahre alt. Ärzte älter als 6o Jahre machten knapp ein Drittel der Studienpopulation aus, während lediglich 3\% jünger als 39 Jahre waren. Der Anteil an weiblichen Teilnehmern lag bei 28\%. Die teilnehmenden Ärzte waren zum Großteil (62\%) Allgemeinmediziner. Die am zweithäufigsten vertretene Fachrichtung war die Innere Medizin mit einem Anteil von 16\%, gefolgt von Urologen mit $6 \%$. Der restliche Anteil verteilte sich auf verschiedene andere Disziplinen wie HNO-Heilkunde, Dermatologie oder Neurochirurgie. Abbildung 21 zeigt die Verteilung der teilnehmenden Ärzte auf die verschiedenen Praxisformen.

Die größte Gruppe von Ärzten (36\%) betreuen pro Tag durchschnittlich mehr als 75 Patienten. 51 bis 75 Patienten pro Tag werden von $28 \%$ der Ärzte betreut,

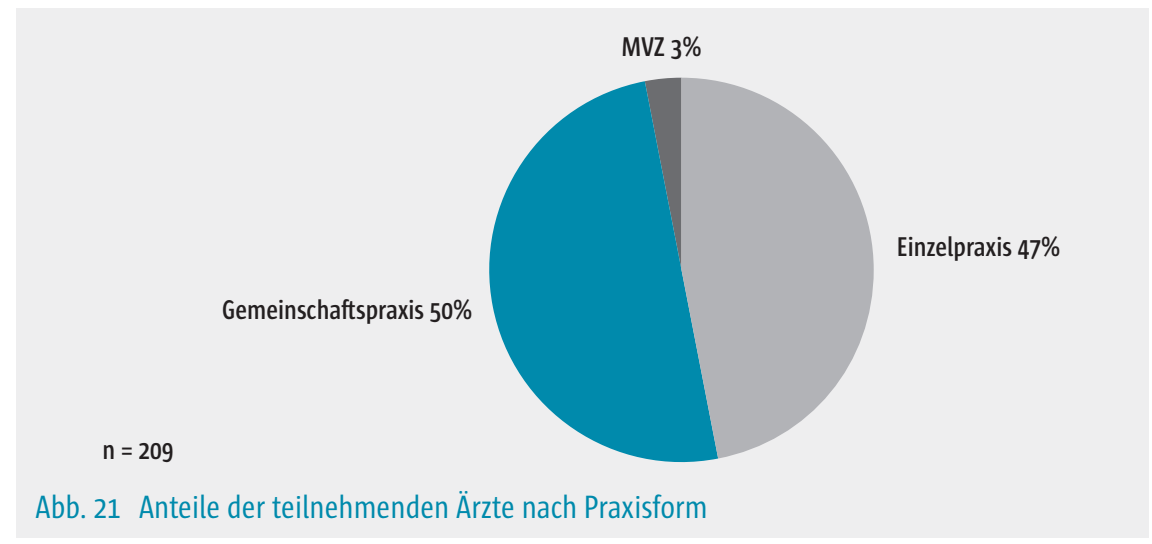




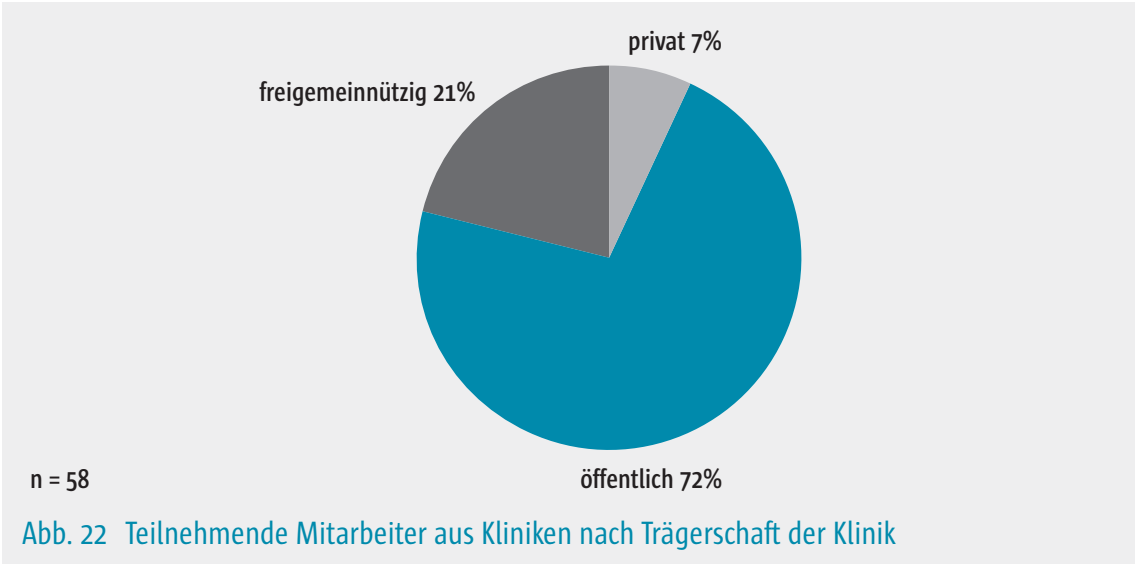

26 bis 50 Patienten von $31 \% .5 \%$ der Befragten betreuen durchschnittlich weniger als 25 Patienten pro Tag.

\subsubsection{Teilnehmer aus dem Klinikbereich}

Unter den Klinikmitarbeitern waren 28\% im Sozialdienst tätig. 13\% gaben an im Bereich Wundversorgung und Ernährungsmanagement zu arbeiten. Weitere $13 \%$ gehörten der Pflegedienstleitung oder der Stationsleitung an. Jeweils 12\% waren als Gesundheits- bzw. Krankenpfleger oder Casemanager tätig. Die restlichen Befragten verteilten sich auf die Disziplinen Arzt (5\%), Qualitätsmanagement $(1,7 \%)$, Angehörigencoach $(1,7 \%)$ und Studienassistentin $(1,7 \%)$. Die Trägerstruktur wird aus Abbildung 22 ersichtlich. Die Mehrheit der Befragten (72\%) arbeitete in Kliniken öffentlicher Trägerschaft.

Jeweils circa 30\% der Befragten vertraten Kliniken mit mehr als 1.000 Betten und 500 bis 1.000 Betten. 16\% der Befragten arbeiten in Häusern mit 300 bis 500 Betten, 21\% in Häusern mit 100 bis 300 Betten und lediglich $2 \%$ in Kliniken mit bis zu 100 Betten. Abbildung 23 zeigt die Verteilung der teilnehmenden Mitarbeiter aus Kliniken nach der jeweiligen Versorgungsstufe der Klinik.

Abbildung 24 veranschaulicht, in welchen Bereichen die in den Kliniken arbeitenden Fachkräfte tätig sind, die nach Angaben der Befragten schwerpunktmäßig für die Homecare-Therapie zuständig ist.

Der Großteil der Befragten gab an, ein für Homecare zuständiges Wundteam in ihrer Klinik zu haben. Aber auch andere Disziplinen wie Ernährungs- und Diabetesteams sowie Stoma-, Schmerz- und Palliativteams sind in der Mehrzahl der vertretenen Kliniken vorhanden. 61\% der Befragten gaben zudem an, dass ihre Klinik über mindestens einen Case-Manager verfügt. Bei 52\% der Befragten sind mehrere Case-Manager in der Klinik angestellt. Ein Sozialdienst ist in den Kliniken aller Befragten vorhanden. 

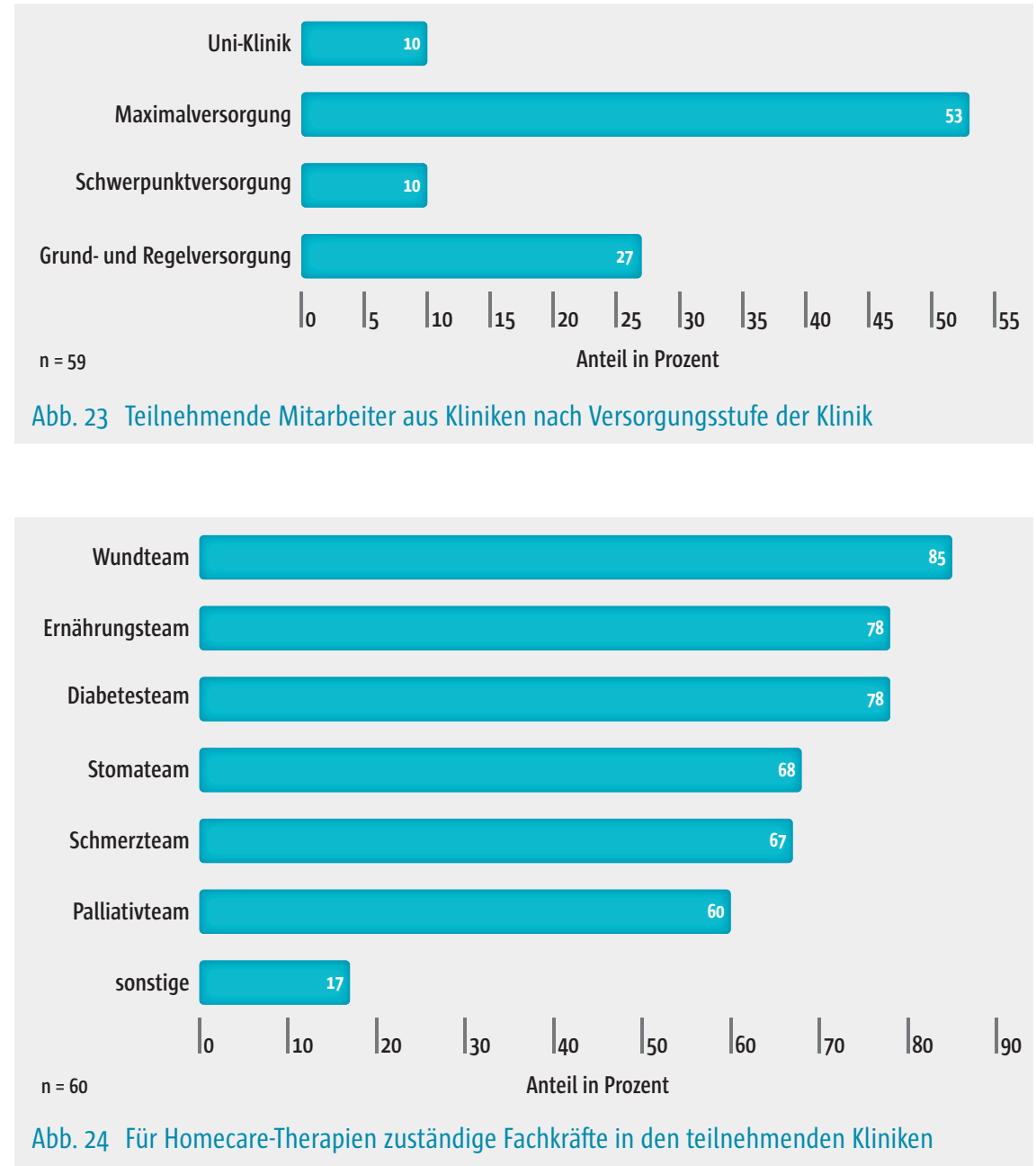

\subsubsection{Teilnehmer aus der stationären Pflege}

Der größte Teil der Mitarbeiter aus stationärer Pflege (74\%) war in der Pflegedienst- oder Wohnbereichsleitung tätig (vgl. Abbildung 25).

Über die Hälfte der befragten Mitarbeiter (58\%) arbeitete in Einrichtungen aus öffentlicher Trägerschaft. $37 \%$ kamen aus freigemeinnützig geführten Einrichtungen und $5 \%$ aus privaten Institutionen. Pflegemitarbeiter aus Einrichtungen mit mehr als 100, 51 bis 100 und bis zu 50 Plätzen waren jeweils zu $32 \%, 52 \%$ und $15 \%$ vertreten. 


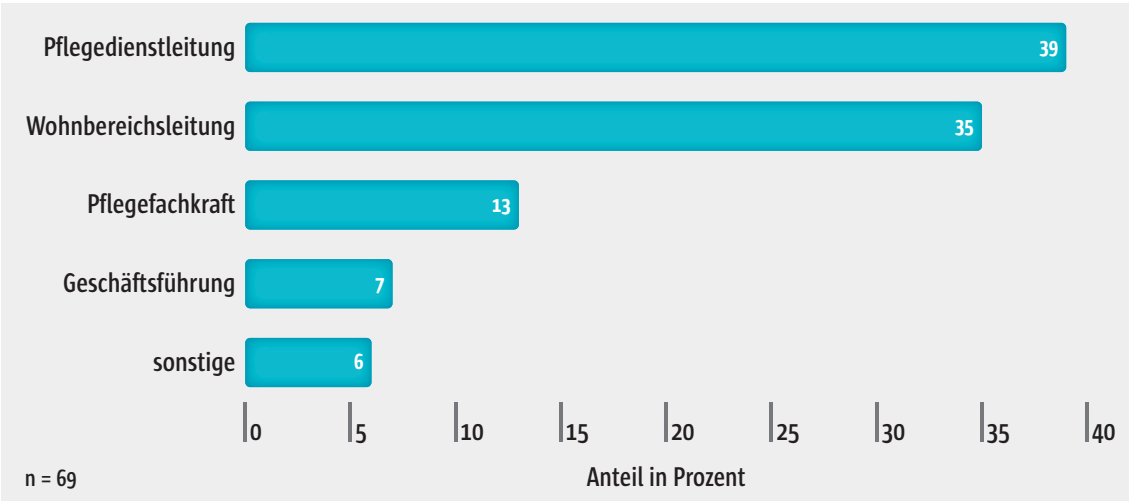

Abb. 25 Funktion der teilnehmenden Mitarbeiter aus stationären Pflegeeinrichtungen

\subsubsection{Teilnehmer aus der ambulanten Pflege}

Im Bereich der ambulanten Pflegedienste kam der überwiegende Teil der Befragten aus der (stellvertretenden) Pflegedienstleitung (70\%) und der Geschäftsführung (21\%). Die übrigen Prozent verteilen sich auf sonstige Funktionen im Bereich der Pflege. Abbildung 26 zeigt die Anteile der Befragten nach der Anzahl der Pflegebedürftigen, die der jeweilige Pflegedienst durchschnittlich betreut.

Die Hälfte der Befragten arbeitete in freigemeinnützigen, $38 \%$ in privaten und $13 \%$ in öffentlich geführten Pflegediensten.

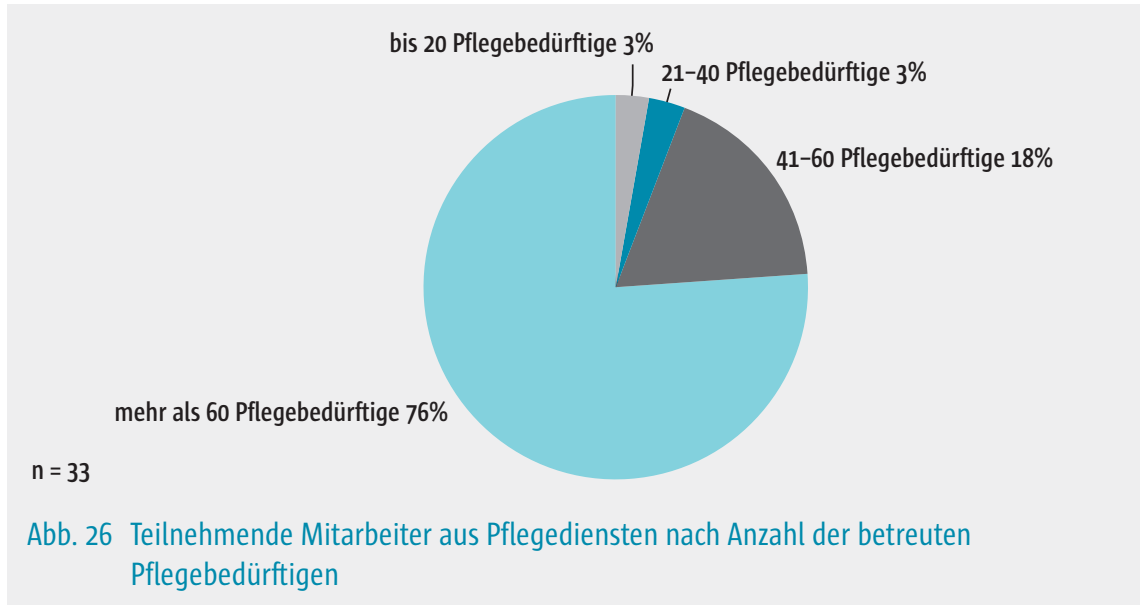




\subsection{Homecare im Versorgungsalltag}

Unter den niedergelassenen Ärzten schätzen 48\% die Bedeutung von Homecare in ihrem Versorgungsalltag als ziemlich bis sehr relevant ein (vgl. Abbildung 27). Unter den übrigen Teilnehmergruppen wird die Relevanz mit Anteilen zwischen 70 und $77 \%$ von deutlich mehr Befragten hoch eingeschätzt. Knapp ein Fünftel der Ärzte sieht wenig bis keine Relevanz in diesem Versorgungsbereich. Im Klinikbereich sind es knapp 12\%. Die Nennungen aus dem Pflegebereich sind vernachlässigbar.

Der unter allen Berufsgruppen am häufigsten genannte Grund für die Bedeutung von Homecare im Versorgungsalltag ist die Komplexität der benötigten Behandlung gefolgt von der Anzahl der betroffenen Patienten bzw. Pflegebedürftigen. Klinikmitarbeiter heben zudem den sozialen Hintergrund des Patienten sowie das erhöhte Risiko eines Drehtüreffekts bei einer komplexen Versorgungslage vor (jeweils 61\%). Die Verantwortung gegenüber dem Patienten ist für 54\% der Klinikmitarbeiter ein Grund für die Bedeutung von Homecare, die Anzahl der betroffenen Patienten für 51\%. Die Auswirkungen auf die Verweildauer bzw. auf das Klinikbudget spielen für 48\% eine Rolle. Für Mitarbeiter aus der stationären Pflege sind der vergleichsweise hohe Koordinationsaufwand mit den behandelnden Ärzten und die Verantwortung gegenüber den Bewohnern mit 45\% bzw. 43\% zusätzlich relevante Gründe. In der ambulanten Pflege werden die zwei zuletzt genannten Gründe zu 36\% und $33 \%$ genannt. $29 \%$ der niedergelassenen Ärzte empfinden ebenso die Auswir-

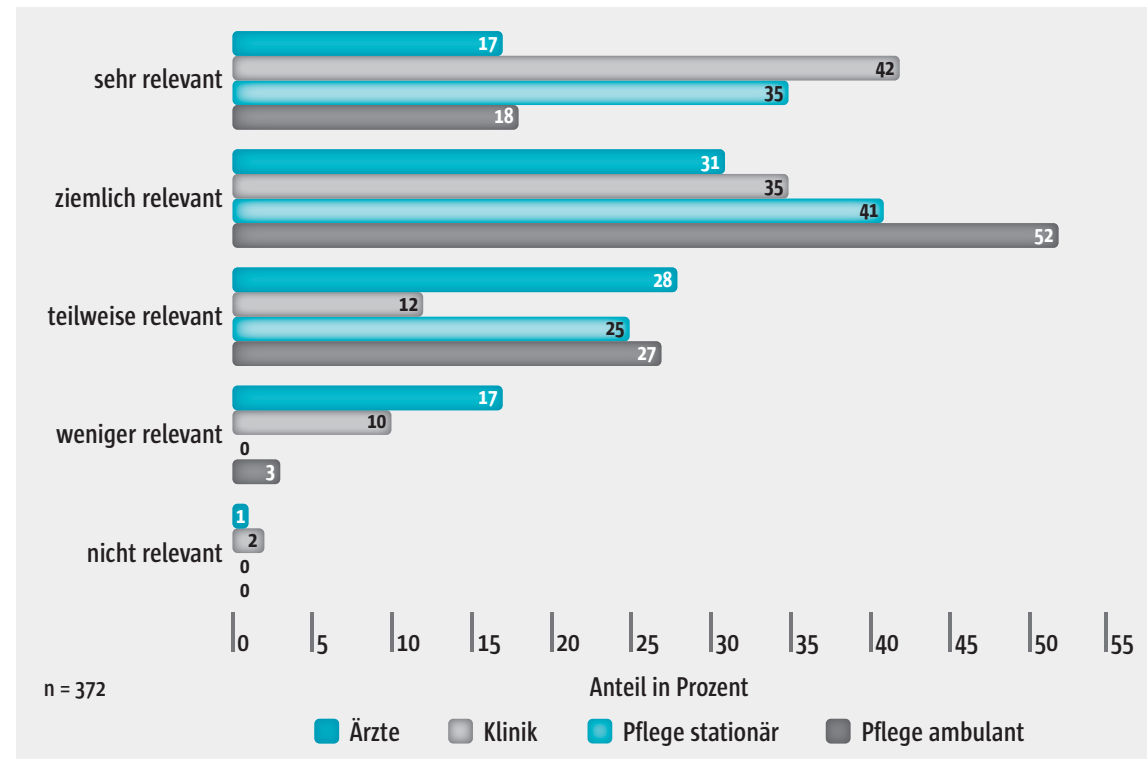

Abb. 27 Relevanz der Homecare-Versorgung für den Versorgungsalltag nach Berufsgruppen 
kungen von Homecare auf ihr Praxisbudget als maßgeblich für dessen Relevanz.

Unter den niedergelassenen Ärzten und den Klinikvertretern fällt die Bewertung der aktuellen Versorgung von Homecare-Patienten im deutschen Gesundheitswesen relativ schlecht aus (vgl. Abbildung 28). Lediglich jeweils $3 \%$ der beiden Berufsgruppen empfinden die aktuelle Versorgung als sehr gut. Der Großteil der Befragten (circa 60\% der Ärzte und 66\% aus Klinik) schätzt die Versorgungssituation von Homecare-Patienten als höchstens befriedigend ein. Im Gegensatz dazu bewerten Mitarbeiter aus den Pflegebereichen die aktuelle Situation wesentlich positiver. In der stationären und ambulanten Pflege wird die Homecare-Versorgung zumeist als sehr gut $(17 \%, 13 \%)$ und gut $(58 \%, 38 \%)$ beurteilt.

Für niedergelassene Ärzte und Vertreter aus stationärer Pflege lässt sich zeigen, dass die Bewertung der aktuellen Versorgung im positiven Zusammenhang mit der Einschätzung der Relevanz der Versorgung steht. Jene Personen, die die aktuelle Versorgung besser bewerten, schätzen deren Relevanz auch höher ein (Ärzte: Ordinalmaß Gamma = 0,171; p-Wert = o,047; $\mathrm{n}=188$; stationäre Pflege: Ordinalmaß Gamma = 0,520; $p$-Wert =o,001; $n=67$ ). Somit liegt die Vermutung nahe, dass schlechte oder mangelnde Erfahrungen mit

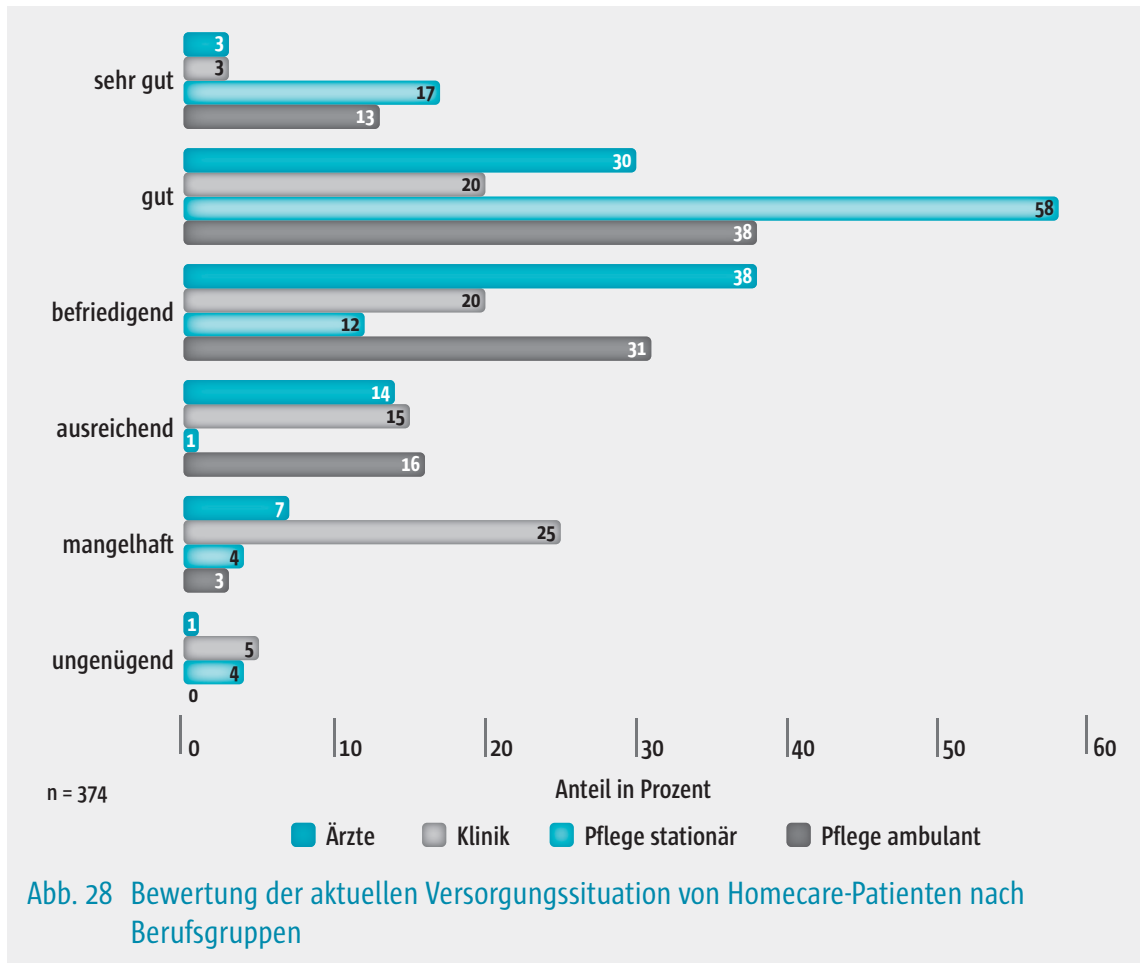


Homecare-Patienten zu einer Unterschätzung der Bedeutung dieses Versorgungsbereiches führen oder umgekehrt. Dieser Zusammenhang lässt sich jedoch nicht für Teilnehmer aus der Klinik sowie der ambulanten Pflege zeigen.

Im Rahmen der Fokusgruppendiskussionen erwies sich aus Sicht der Teilnehmer die Auswahl eines Homecare-Dienstleisters als besonders kritisch und wurde damit in die schriftliche Befragung aufgenommen. Auch in der schriftlichen Befragung zeigt sich hier eine relativ große Meinungsverschiedenheit zwischen den einzelnen Berufsgruppen. Nach Angaben der Ärzte erfolgt die Auswahl und Information eines Homecare-Dienstleisters zu einem weiten Prozentsatz vornehmlich durch die Klinik (67\%). Dies deckt sich zunächst mit den Ansichten der Mitarbeiter aus der Klinik, die angeben in 51\% der Fälle, den Homecare-Dienstleister zu wählen. Während 45\% der Ärzte angeben, dass sie selbst den Homecare-Dienstleister kontaktieren, bestätigen das Mitarbeiter aus der Klinik jedoch nur in 21\% der Fälle. Die Kliniken heben im Vergleich dazu die Rolle von Angehörigen mehr hervor. 26\% der teilnehmenden Klinikmitarbeiter sehen die Entscheidung bei den Angehörigen, wobei es nur $2 \%$ der niedergelassenen Ärzte so sehen. Mitarbeiter aus stationärer und ambulanter Pflege geben an, zu 97\% bzw. 76\% verantwortlich zu sein für die Ansprache eines Homecare-Dienstleisters, sofern notwendig. Während die Mitarbeiter aus ambulanter Pflege zu einem gleichen Prozentsatz auch angeben, dass die Ansprache durch die Klinik erfolgt, sind es in der stationären Pflege nur 18\% der Befragten. Lediglich 17-24\% beider Berufsgruppen gibt an, dass dies durch den niedergelassenen Arzt erfolgt. Mehrfachantworten bei allen Berufsgruppen deuten insgesamt darauf hin, dass die Entscheidung häufig von verschiedenen Akteuren getroffen wird und klare Verantwortlichkeiten zu fehlen scheinen.

\subsection{Zusammenarbeit mit externen Homecare-Dienstleistern}

Zum Zeitpunkt der Umfrage arbeiteten 74\% der niedergelassenen Ärzte mit Homecare-Fachkräften zusammen, die nicht bei ihnen angestellt sind. Von diesen planten 6\% die Zusammenarbeit in Zukunft auszubauen. Etwas mehr als die Hälfte der befragten Ärzte arbeitete mit mehr als einem Homecare-Dienstleister zusammen. Von den $26 \%$ der Ärzte, die zum Befragungszeitpunkt nicht mit externen Homecare-Fachkräften zusammenarbeiteten, planten lediglich $4 \%$ dies in der Zukunft zu ändern. Dabei lässt sich kein Zusammenhang der Kooperationsbereitschaft der Ärzte mit deren Alter feststellen.

Die Zusammenarbeit mit dem Homecare-Dienstleister wurde von 56\% der Ärzte als gut oder sehr gut bewertet - 19\% halten sie für befriedigend, nur $5 \%$ empfinden sie als ungenügend oder mangelhaft. Abbildung 29 zeigt eine 


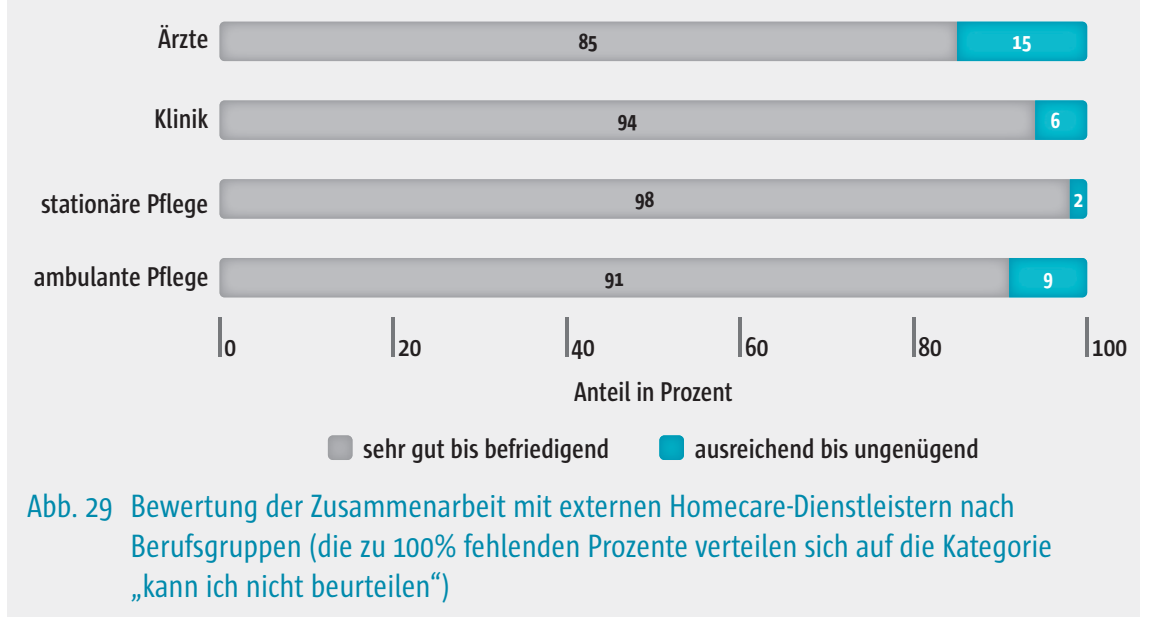

Übersicht der Bewertung der Zusammenarbeit mit externen HomecareDienstleistern der einzelnen Berufsgruppen.

Die Bewertung fällt bei allen Berufsgruppen sehr positiv aus. Nur ein kleiner Bruchteil der Befragten je Berufsgruppe empfindet die Zusammenarbeit mit dem Homecare-Dienstleister schlechter als befriedigend. Unter den Ärzten sind noch die meisten Personen zu finden, die unzufrieden sind (13\%). Im Vergleich mit der Bewertung der Zusammenarbeit zwischen den Berufsgruppen untereinander, schneidet der Homecare-Dienstleister insgesamt ebenfalls am besten ab. Die Bewertung der Zusammenarbeit der Ärzte mit dem Homecare-Dienstleister steht dabei in Zusammenhang mit der Auswahl des Dienstleisters (Nominalmaß Cramer-V = 0,265; p-Wert = o, 017; $\mathrm{n}=171$ ).

Der Großteil der Befragten aller Berufsgruppen arbeitet mit mehr als einem Homecare-Dienstleister zusammen: 51\% der Ärzte, 54\% der Klinikmitarbeiter, 35\% der Mitarbeiter aus stationärer Pflege und 67\% der Mitarbeiter aus ambulanter Pflege. Insgesamt sind die Befragten mit der Zusammenarbeit ebenso zufrieden, auch wenn die Bewertung geringfügig schlechter ausfällt.

Die niedergelassenen Ärzte wurden zudem nach den Leistungen befragt, die sie derzeit von externen Homecare-Dienstleistern in Anspruch nehmen. Unter denen, die mit externen Homecare-Dienstleistern zusammenarbeiten, zählen die meisten Ärzte Leistungen wie indikationsbezogene und leitlinienkonforme Therapievorschläge, Rückmeldung zur Therapieanpassung sowie eine verbesserte Kommunikation und Koordination zwischen den Beteiligten voll oder teilweise zu den Leistungen ihres Homecare-Dienstleisters (vgl. Abbildung 30).

Ein gemischtes Bild ergibt sich bei der Bereitstellung therapierelevanter Diagnosen und Befunde rechtzeitig vor Entlassung des Patienten aus dem Kran- 


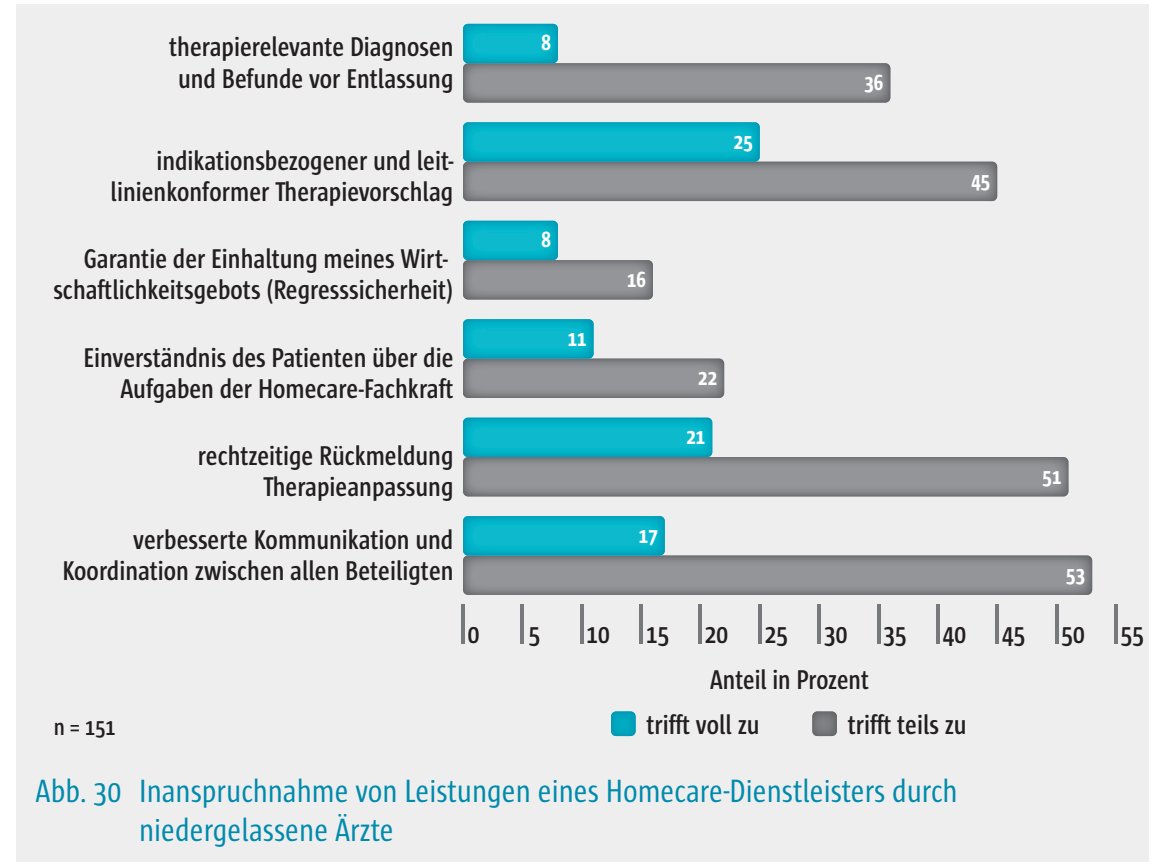

kenhaus. Dies wird von 43\% der Ärzte ganz oder teilweise zu den Leistungen ihres Homecare-Dienstleisters gezählt. Eine eindeutige Mehrheit $(76 \%$ und $67 \%$ ) sieht die Garantie des Homecare-Dienstleisters gegenüber dem Arzt zur Einhaltung des Wirtschaftlichkeitsgebotes und der Vorbeugung von Regress bei der Produktauswahl sowie das Einholen eines schriftlichen Einverständnisses des Patienten über die Tätigkeitsbefugnisse der Homecare-Fachkraft derzeit nicht als Aufgabe ihres Homecare-Dienstleisters.

Die Daten zeigen, dass die Inanspruchnahme von Homecare-Leistungen und die Bewertung der aktuellen Versorgung von Homecare-Patienten voneinander abhängig sind. Die Bereitstellung indikationsbezogener Therapievorschläge, Garantien zur Einhaltung des Wirtschaftlichkeitsgebots sowie die rechtzeitige Rückmeldung zur Therapieanpassung stehen in stark positivem Zusammenhang mit der positiven Bewertung der aktuellen Versorgungsituation durch die Ärzte (vgl. Tabelle 7).

\subsection{Zusammenarbeit mit anderen Berufsgruppen}

Die Zusammenarbeit zwischen den einzelnen Berufsgruppen im Rahmen der Homecare-Versorgung wird ebenfalls überwiegend als gut bewertet, wenn auch mit leichten Abstufungen. Abbildung 31 zeigt die gegenseitige Bewertung der Zusammenarbeit zwischen Klinik und Pflege, die von der Mehrheit auf beiden Seiten positiv bewertet wird. Die Zusammenarbeit zwischen Kli- 
Tab. 7 Zusammenhang zwischen Leistungsinanspruchnahme und Bewertung der HomecareVersorgung ( $n=135$ bis 143 )

\begin{tabular}{ll} 
Leistungen des Homecare-Dienstleisters & Bewertung der Homecare- Versorgung \\
& Gamma $(\mathrm{p}$-Wert) \\
\hline Therapierelevante Diagnosen und Befunde & $0,170(0,163)$ \\
\hline Indikationsbezogener Therapievorschlag & $0,383(0,000)^{* * *}$ \\
\hline Garantie der Einhaltung des Wirtschaftlichkeitsgebotes & $0,408(0,004)^{* *}$ \\
\hline Einverständnis des Patienten & $0,098(0,471)$ \\
\hline Rückmeldung zur Therapieanpassung & $0,263(0,021)^{*}$ \\
\hline Kommunikation und Koordination der Beteiligten & $0,175(0,166)$ \\
\hline$* * * p-W e r t \leq 0,001,{ }^{* *}$ p-Wert $\leq 0,005,{ }^{*}$ p-Wert $\leq 0,05$ & \\
\hline
\end{tabular}

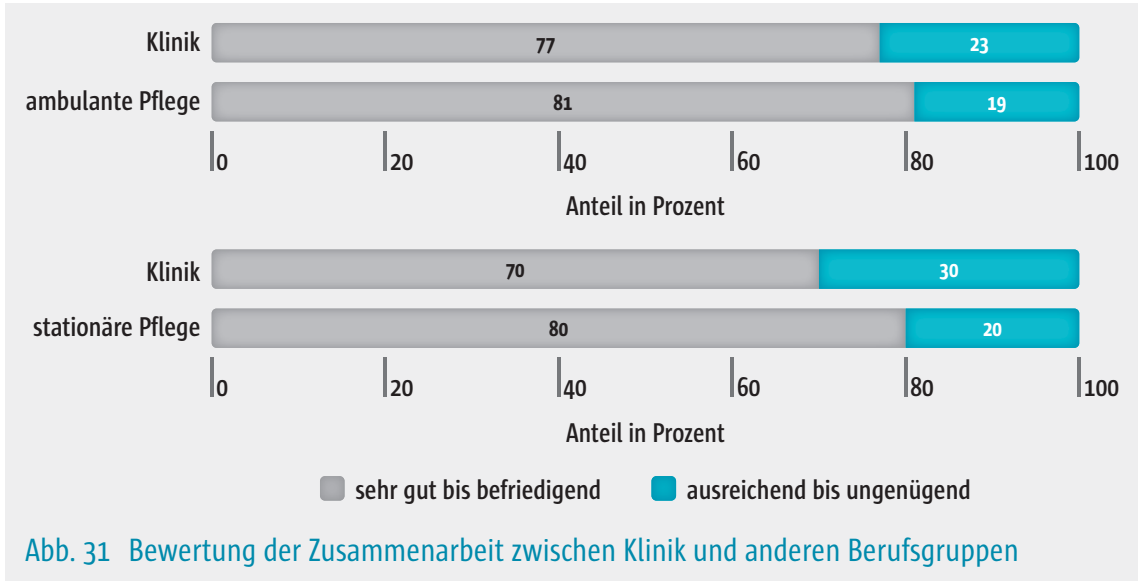

nik und ambulanter Pflege scheint etwas besser zu funktionieren. Erstaunlich ist die dichte Übereinstimmung der Ansichten der jeweiligen Berufsgruppen - 70\% der Klinikmitarbeiter bewerten die Zusammenarbeit mit der ambulanten Pflege als gut genauso wie $76 \%$ der Vertreter in der ambulanten Pflege.

Etwas größere Diskrepanzen gibt es bei der Einschätzung der Zusammenarbeit zwischen Ärzten und anderen Berufsgruppen wie aus Abbildung 32 ersichtlich wird. Die größte Diskrepanz besteht zwischen den Ansichten der Ärzte und der ambulanten Pflege. Dennoch wird insgesamt die Zusammenarbeit zwischen Ärzten und anderen Berufsgruppen überwiegend positiv bewertet. Den größten Anteil negativer Bewertungen erhalten die Ärzte von Mitarbeitern aus dem Klinikbereich. Jeweils 27\% schätzen die Zusammen- 

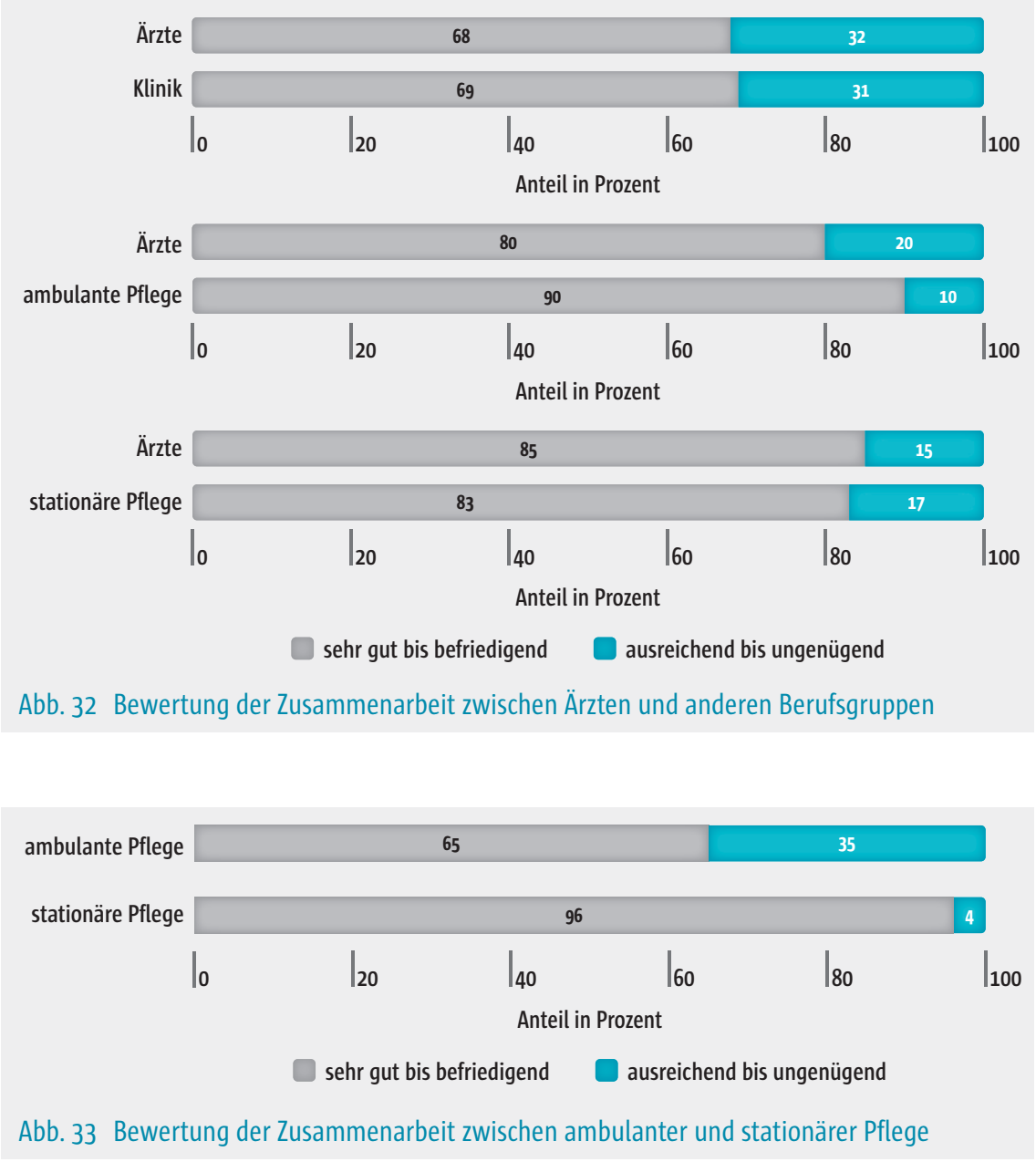

arbeit nicht mehr als ausreichend ein. 60\% der Klinikmitarbeiter empfinden die Zusammenarbeit mit Ärzten hingegen sehr gut bis befriedigend.

Am auffälligsten zeigt sich die Einschätzung der Zusammenarbeit der beiden Pflegebereiche untereinander (vgl. Abbildung 33). Hierbei ist darauf zu achten, dass etwa die Hälfte der jeweiligen Teilnehmer, keine Bewertung abgegeben hat. Dies könnte darauf hinweisen, dass ambulante und stationäre Pflege bislang nicht unmittelbar miteinander zusammenarbeiten. 36\% der Teilnehmer aus dem Bereich der ambulanten Pflege bewerten die Zusammenarbeit zwischen ambulanter und stationärer Pflege mit sehr gut bis befriedigend. Mit 49\% scheint die stationäre Pflege wesentlich zufriedener mit der Zusammenarbeit. 
Die Zusammenarbeit mit Hilfsmittelversorgern wurde ebenfalls abgefragt und überwiegend als positiv bewertet. Es zeigt sich, dass Ärzte, die mehr Leistungen von Homecare-Anbietern in Anspruch nehmen, die Zusammenarbeit mit Akteuren im Rahmen der Homecare-Versorgung grundsätzlich positiver bewerten - dies gilt insbesondere für die Bewertung der Zusammenarbeit mit den Homecare-Dienstleistern. Tabelle 8 zeigt sämtliche signifikante Zusammenhänge.

Für Ärzte haben insbesondere die verbesserte Kommunikation und Koordination, die rechtzeitige Rückmeldung zur Therapieanpassung sowie die Garantie zur Einhaltung des Wirtschaftlichkeitsgebotes einen positiven Einfluss auf die Zufriedenheit mit der Zusammenarbeit. Tabelle 8 zeigt weiterhin, dass die Inanspruchnahme von Leistungen eines Homecare-Dienstleisters auch zur Zufriedenheit der Ärzte mit der Zusammenarbeit mit anderen Berufsgruppen führen kann. Dies ergibt sich aus den signifikanten Zusammenhängen in den letzten drei Spalten. Den stärksten Effekt hat dabei die Garantie des Wirtschaftlichkeitsgebotes auf die Zusammenarbeit mit dem Hilfsmittellieferant, was unmittelbar intuitiv erscheint.

Tab. 8 Zusammenhang zwischen der Inanspruchnahme von Homecare-Dienstleistungen und der Bewertung der Zusammenarbeit mit verschiedenen Akteuren ( $n=129$ bis 145)

\begin{tabular}{|c|c|c|c|c|c|}
\hline $\begin{array}{l}\text { Inanspruchnahme } \\
\text { von Leistungen }\end{array}$ & $\begin{array}{l}\text { 1. Homecare- } \\
\text { Dienstleister } \\
\text { Gamma } \\
\text { (p-Wert) }\end{array}$ & $\begin{array}{l}\text { 2. Homecare- } \\
\text { Dienst- } \\
\text { leister**** } \\
\text { Gamma } \\
\text { (p-Wert) }\end{array}$ & $\begin{array}{l}\text { Hilfsmittelver- } \\
\text { sorger } \\
\text { Gamma } \\
\text { (p-Wert) }\end{array}$ & $\begin{array}{l}\text { Ambulante } \\
\text { Pflege } \\
\text { Gamma } \\
\text { (p-Wert) }\end{array}$ & $\begin{array}{l}\text { Klinik } \\
\text { Gamma } \\
\text { (p-Wert) }\end{array}$ \\
\hline $\begin{array}{l}\text { Therapierelevante } \\
\text { Diagnosen und } \\
\text { Befunde }\end{array}$ & $\begin{array}{l}0,258 \\
(0,040)^{*}\end{array}$ & $\begin{array}{l}0,439 \\
(0,004)^{* *}\end{array}$ & $\begin{array}{l}0,460 \\
(0,000)^{* * *}\end{array}$ & - & $\begin{array}{c}0,262 \\
(0,017)^{*}\end{array}$ \\
\hline $\begin{array}{l}\text { Indikations- } \\
\text { bezogener } \\
\text { Therapievorschlag }\end{array}$ & $\begin{array}{l}0,345 \\
(0,002)^{* *}\end{array}$ & $\begin{array}{l}0,579 \\
(0,000)^{* * *}\end{array}$ & $\begin{array}{c}0,274 \\
(0,013)^{*}\end{array}$ & $\begin{array}{c}0,343 \\
(0,006)^{*}\end{array}$ & - \\
\hline $\begin{array}{l}\text { Garantie der Einhal- } \\
\text { tung des Wirtschaft- } \\
\text { lichkeitsgebotes }\end{array}$ & $\begin{array}{l}0,444 \\
(0,002)^{* * *}\end{array}$ & - & $\begin{array}{l}0,532 \\
(0,000)^{* * *}\end{array}$ & - & - \\
\hline $\begin{array}{l}\text { Einverständnis } \\
\text { des Patienten }\end{array}$ & $\begin{array}{l}0,288 \\
(0,040)^{*}\end{array}$ & $\begin{array}{c}0,413 \\
(0,016)^{*}\end{array}$ & $\begin{array}{l}0,447 \\
(0,000)^{* * *}\end{array}$ & - & - \\
\hline $\begin{array}{l}\text { Rückmeldung zur } \\
\text { Therapieanpassung }\end{array}$ & $\begin{array}{l}0,631 \\
(0,000)^{* * *}\end{array}$ & $\begin{array}{c}0,526 \\
(0,000)^{* * *}\end{array}$ & - & - & - \\
\hline $\begin{array}{l}\text { Kommunikation und } \\
\text { Koordination der } \\
\text { Beteiligten }\end{array}$ & $\begin{array}{l}0,567 \\
(0,000)^{* * *}\end{array}$ & $\begin{array}{l}0,448 \\
(0,002)^{* *}\end{array}$ & $\begin{array}{l}0,434 \\
(0,000)^{* * *}\end{array}$ & $\begin{array}{l}0,376 \\
(0,002)^{* *}\end{array}$ & - \\
\hline$* * * * n=84$ bis $88, * * * p$ & -Wert $\leq 0,001$, * & Wert $\leq 0,005$ & Wert $\leq 0,05$ & & \\
\hline
\end{tabular}




\subsection{Potentiale der Zusammenarbeit mit externen Homecare-Dienstleistern}

\subsubsection{Einschätzung des Potentials durch niedergelassene Ärzte}

Ärzte sehen das größte Potential in der Zusammenarbeit mit externen Homecare-Dienstleistern im Vorhandensein eines Ansprechpartners (62\%), in der Zeitersparnis (58\%) sowie dem positiven Einfluss auf die Qualität (56\%) (vgl. Abbildung 34). Hingegen erwartet die Mehrheit der Ärzte keine Aufwandsersparnis bei der Qualifikation eigener Mitarbeiter und auch keine Verbesserung der eigenen Reputation durch die Zusammenarbeit mit einem externen Homecare-Dienstleister. Ein sehr gemischtes Bild ergibt sich bei der Einschätzung hinsichtlich einer verstärkten Regresssicherheit durch einen Homecare-Dienstleister: Während 19\% der Ärzte glauben, dass die Regresssicherheit durch eine Zusammenarbeit verbessert werden kann, sehen $54 \%$ hierin nur ein geringes bzw. kein Potential.

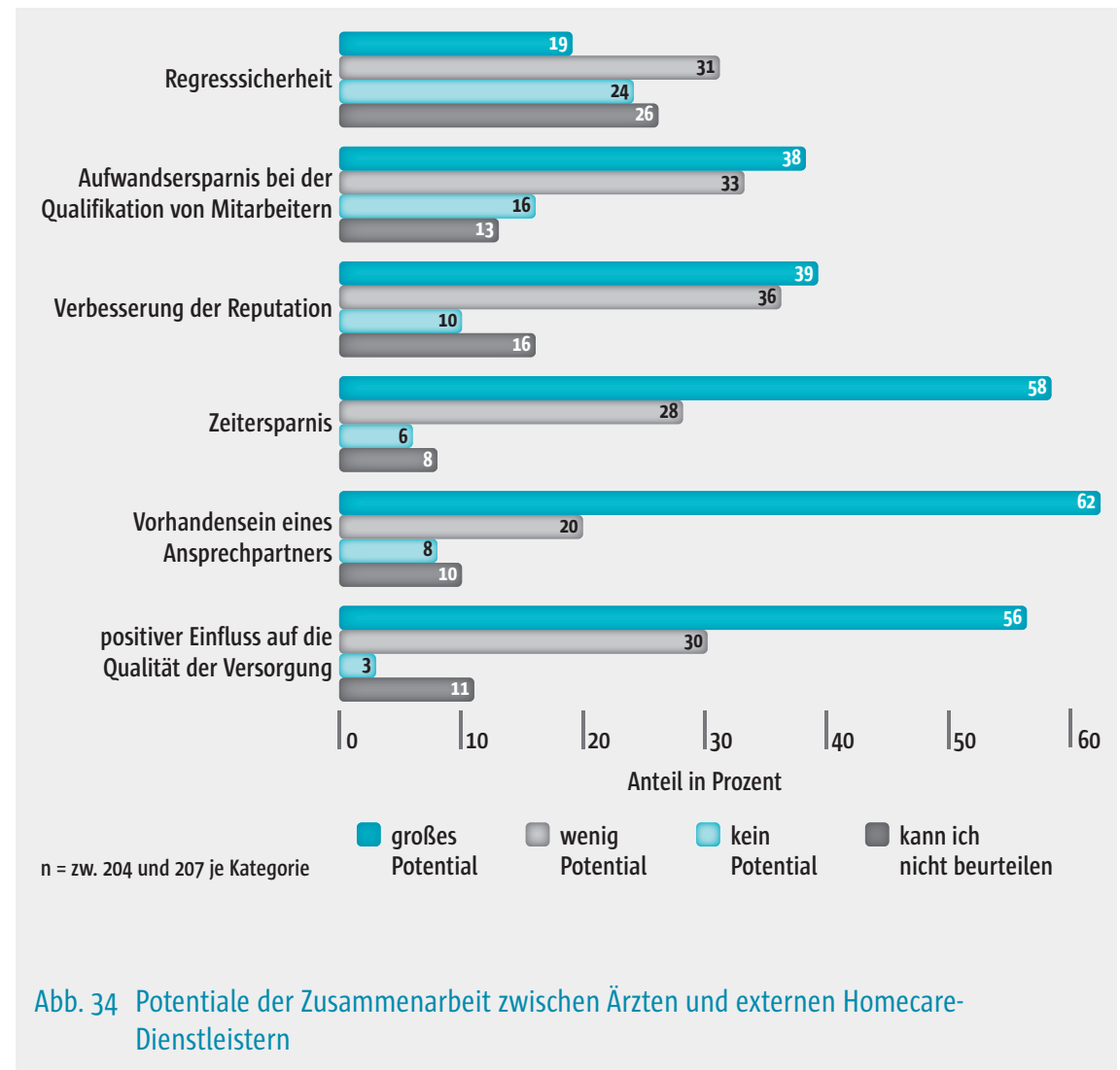


Tab. 9 Zusammenhang zwischen den Potentialen der Zusammenarbeit mit externen Homecare-Dienstleistern und der Relevanz von Homecare im Versorgungsalltag $(\mathrm{n}=174$ bis 187$)$

\begin{tabular}{|c|c|}
\hline $\begin{array}{l}\text { Potentiale der Zusammenarbeit mit externen } \\
\text { Homecare-Dienstleistern }\end{array}$ & $\begin{array}{l}\text { Relevanz von Homecare im Versorgungsalltag } \\
\text { Gamma } \\
\text { (p-Wert) }\end{array}$ \\
\hline Regresssicherheit**** & $0,428(0,000)^{* * *}$ \\
\hline $\begin{array}{l}\text { Aufwandsersparnis bei der Qualifikation von } \\
\text { Mitarbeitern }\end{array}$ & $0,236(0,011)^{*}$ \\
\hline Verbesserung der Reputation & $0,348(0,000)^{* * *}$ \\
\hline Zeitersparnis & $0,324(0,002)^{* *}$ \\
\hline Vorhandensein eines Ansprechpartners & $0,368(0,001)^{* * *}$ \\
\hline Positiver Einfluss auf die Qualität der Versorgung & $0,444(0,000)^{* * *}$ \\
\hline \multicolumn{2}{|c|}{$* * * * n=148,{ }^{* * *} p$-Wert $\leq 0,001,{ }^{* *}$ p-Wert $\leq 0,005,{ }^{*} p$-Wert $\leq 0,05$} \\
\hline
\end{tabular}

Interessant ist ein eindeutiger Zusammenhang zwischen der Einschätzung der Potentiale in der Zusammenarbeit mit externen Homecare-Dienstleistern und der empfundenen Relevanz von Homecare im Versorgungsalltag. Aus Tabelle 9 wird ersichtlich, dass Ärzte, die Homecare als relevant erachten, auch tendenziell ein großes Potential in der Zusammenarbeit mit externen Homecare-Dienstleistern sehen.

Auch die Inanspruchnahme von Homecare-Leistungen steht in Zusammenhang mit der Einschätzung der Potentiale der Zusammenarbeit durch die Ärzte. Tabelle 10 stellt die signifikanten Zusammenhänge dar.

Je höher das Potential der Zusammenarbeit eingeschätzt wird, desto eher werden entsprechende Leistungen eines Homecare-Anbieters in Anspruch genommen. Am stärksten zeigt sich der Zusammenhang im Potential eines Ansprechpartners mit der Inanspruchnahme der Rückmeldung zur Therapieanpassung. Ärzte, die von ihrem Homecare-Anbieter über die Änderung von Befunden und Therapien informiert werden, sehen im Vorhandensein eines festen Ansprechpartners eher ein großes Potential, als Ärzte, die diese Leistungen nicht erhalten. Erfahrungen in der Inanspruchnahme von Leistungen führen demnach zu positiven Einschätzungen bzgl. der HomecarePotentiale - und umgekehrt fördern positive Erwartungen an die Zusammenarbeit wiederum die Nutzung von Homecare-Dienstleistungen. Ein noch deutlicheres Bild zeigt sich bei der Gegenüberstellung von den erwarteten Potentialen und den tatsächlichen Erfahrungen der Ärzte in der Zusammenarbeit mit externen Homecare-Dienstleistern. Insbesondere sehen die Ärzte großes Potential im Vorhandensein eines Ansprechpartners, in der Zeitersparnis sowie auch in der Verbesserung der eigenen Reputation, die posi- 
Tab. 10 Zusammenhänge zwischen den Potentialen der Zusammenarbeit mit externen Homecare-Dienstleistern und der Inanspruchnahme von Homecare-Dienstleistungen ( $n=110$ bis 144)

\begin{tabular}{|c|c|c|c|c|c|c|}
\hline \multirow{3}{*}{$\begin{array}{l}\text { Potentiale der } \\
\text { Zusammen- } \\
\text { arbeit }\end{array}$} & \multicolumn{6}{|c|}{ In Anspruch genommene Homecare-Dienstleistung } \\
\hline & $\begin{array}{l}\text { Diagno- } \\
\text { sen/Be- } \\
\text { funde }\end{array}$ & $\begin{array}{l}\text { Therapie- } \\
\text { vorschlag }\end{array}$ & $\begin{array}{l}\text { Wirtschaft- } \\
\text { lichkeitsga- } \\
\text { rantie }\end{array}$ & $\begin{array}{l}\text { Emp- } \\
\text { fang von } \\
\text { Rezepten }\end{array}$ & $\begin{array}{l}\text { Therapie- } \\
\text { anpassung }\end{array}$ & $\begin{array}{l}\text { Kommuni- } \\
\text { kation }\end{array}$ \\
\hline & $\begin{array}{l}\text { Gamma } \\
\text { (p-Wert) }\end{array}$ & $\begin{array}{l}\text { Gamma } \\
\text { (p-Wert) }\end{array}$ & $\begin{array}{l}\text { Gamma } \\
\text { (p-Wert) }\end{array}$ & $\begin{array}{l}\text { Gamma } \\
\text { (p-Wert) }\end{array}$ & $\begin{array}{l}\text { Gamma } \\
\text { (p-Wert) }\end{array}$ & $\begin{array}{l}\text { Gamma } \\
\text { (p-Wert) }\end{array}$ \\
\hline $\begin{array}{l}\text { Steigerung der } \\
\text { Versorgungs- } \\
\text { qualität }\end{array}$ & - & - & - & - & $\begin{array}{c}0,333 \\
(0,022)^{*}\end{array}$ & - \\
\hline $\begin{array}{l}\text { Ansprech- } \\
\text { partner }\end{array}$ & $\begin{array}{c}0,341 \\
(0,026)^{*}\end{array}$ & $\begin{array}{l}0,418 \\
(0,002)^{* *}\end{array}$ & $\begin{array}{c}0,382 \\
(0,036)^{*}\end{array}$ & - & $\begin{array}{c}0,458 \\
(0,001)^{* * *}\end{array}$ & $\begin{array}{c}0,298 \\
(0,048)^{*}\end{array}$ \\
\hline Zeitersparnis & - & $\begin{array}{c}0,270 \\
(0,049)^{*}\end{array}$ & - & - & $\begin{array}{c}0,347 \\
(0,010)^{*}\end{array}$ & $\begin{array}{l}0,297 \\
(0,030)^{*}\end{array}$ \\
\hline $\begin{array}{l}\text { Verbesserte } \\
\text { Reputation }\end{array}$ & - & - & $\begin{array}{c}0,375 \\
(0,017)^{*}\end{array}$ & $\begin{array}{c}0,304 \\
(0,036)^{*}\end{array}$ & $\begin{array}{c}0,300 \\
(0,020)^{*}\end{array}$ & $\begin{array}{l}0,394 \\
(0,003)^{* *}\end{array}$ \\
\hline $\begin{array}{l}\text { Aufwands- } \\
\text { ersparnis bei } \\
\text { Mitarbeiter- } \\
\text { qualifikation }\end{array}$ & - & - & - & - & $\begin{array}{c}0,338 \\
(0,009)^{*}\end{array}$ & - \\
\hline $\begin{array}{l}\text { Regress- } \\
\text { sicherheit }\end{array}$ & $\begin{array}{c}0,324 \\
(0,025)^{*}\end{array}$ & - & $\begin{array}{c}0,489 \\
(0,002)^{* *}\end{array}$ & $\begin{array}{l}0,426 \\
(0,005)^{* *}\end{array}$ & - & - \\
\hline$* * *$ p-Wert $\leq 0,00$ & $*$ p-Wert $\leq$ & $5, *$ p-Wert & & & & \\
\hline
\end{tabular}

tivere Erfahrungen mit ihrem Homecare-Dienstleister gemacht haben, als jene Ärzte, die hier geringere oder keine Potentiale sehen (vgl. Tabelle 11).

\subsubsection{Einschätzung des Potentials durch Vertreter anderer Berufsgruppen}

Im Vergleich zu den Ärzten sehen weit mehr Klinikmitarbeiter große Potentiale in der Zusammenarbeit mit externen Homecare-Dienstleistern (vgl. Abbildung 35). Hierbei spielen das Vorhandensein eines Ansprechpartners, ein positiver Einfluss auf die Qualität der Versorgung sowie die Erhöhung der Patientenzufriedenheit eine Rolle. Auch eine Zeitersparnis und Entlastung des Personals sowie die Verbesserung der Klinikreputation und die Möglichkeit eines geregelten Haftungsübergangs sehen mindestens 50\% der Befragten als große Potentiale. 
Tab. 11 Zusammenhang zwischen den Potentialen der Zusammenarbeit mit externen Homecare-Dienstleistern und der Bewertung der Zusammenarbeit mit dem ersten Homecare-Dienstleister ( $n=135$ bis 168 )

\begin{tabular}{|c|c|}
\hline Potentiale der Zusammenarbeit & $\begin{array}{l}\text { 1. Homecare-Dienstleister } \\
\text { Gamma } \\
\text { (p-Wert) }\end{array}$ \\
\hline Steigerung der Versorgungsqualität & $0,565(0,000)^{* * *}$ \\
\hline Ansprechpartner & $0,436(0,000)^{* * *}$ \\
\hline Zeitersparnis & $0,381(0,001)^{* * *}$ \\
\hline Verbesserte Reputation & $0,397(0,000)^{* * *}$ \\
\hline Aufwandsersparnis bei Mitarbeiterqualifikation & $0,249(0,026)^{*}$ \\
\hline Regresssicherheit & $0,241(0,048)^{*}$ \\
\hline${ }^{* * *}$ - -Wert $\leq 0,001,{ }^{* *}$ p-Wert $\leq 0,005,{ }^{*} p$-Wert $\leq 0,05$ & \\
\hline
\end{tabular}

In diesem Zusammenhang wurden die Klinikmitarbeiter auch dazu befragt, welches Potential die Zusammenarbeit mit externen Homecare-Dienstleistern für die Beziehungsqualität mit den zuweisenden Ärzten der Klinik haben könnte. Croße Potentiale werden hierbei insbesondere in der umgehenden Weiterleitung aller therapierelevanten Daten an den weiterbehandelnden Arzt (70\%) und der Funktion der Homecare-Fachkraft als koordinierende Therapie-Assistenz zwischen Klinik und hausärztlicher Versorgung (68\%) gesehen. Der Ausbau und die Verbesserung der Kommunikation auch beim Zuweisermanagement werden von 61\% der Klinikmitarbeiter als großes Potential gesehen.

Aus Sicht der stationären und ambulanten Pflege zeichnet sich ein ähnlich positives Bild ab. Die Mehrheit der Befragten sieht in der Zusammenarbeit mit externen Homecare-Dienstleistern großes Potential für die Produktversorgung. Leistungen wie die ausführliche Einweisung und Schulung des Pflegepersonals bezüglich der Anwendung der Produkte und Materialien, Rezeptmanagement, termingerechte und zuverlässige Lieferung von Produkten sowie das Vorhandensein eines schriftlichen Therapieplans werden von den meisten positiv bewertet. Neben den Potentialen für die Produktversorgung wurden die Mitarbeiter der Pflegeinrichtungen auch nach den Potentialen für die organisatorischen und behandlungspflegerischen Tätigkeiten bei Homecare-Patienten mit komplexer Versorgungssituation befragt. Auch hier sehen viele Befragte große Potentiale. Sie liegen insbesondere im Vorhandensein eines Ansprechpartners, in der Durchführung von Fortbildungen und schulungen zu medizinischen und produktspezifischen Themen sowie in bedarfsgerechten und regelmäßig abgestimmten Therapievisiten. Am wenigsten Potential wird dem Aspekt der Aufwandsersparnis durch 


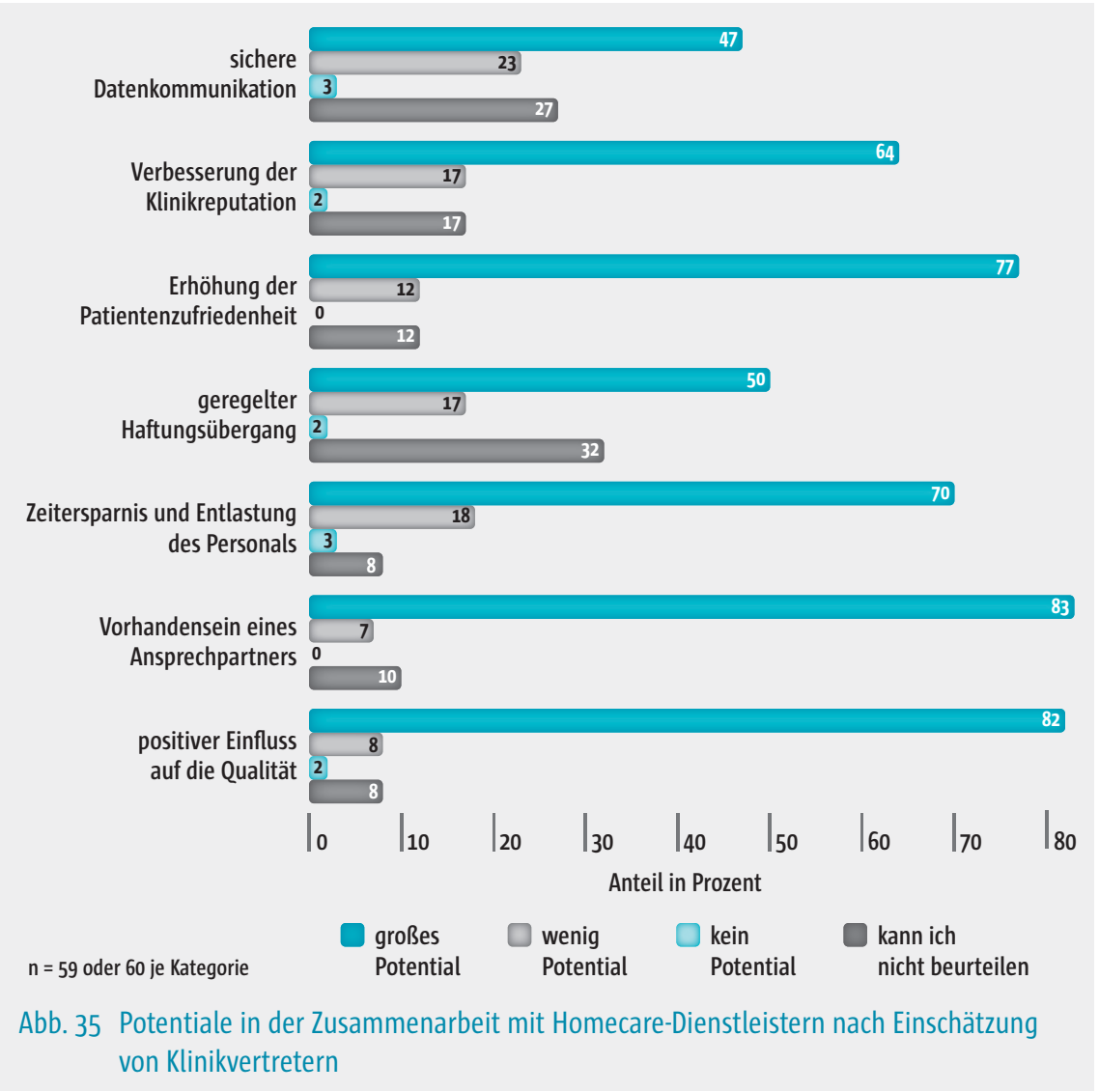

die Zusammenarbeit mit externen Homecare-Dienstleistern zugesprochen. Lediglich $37 \%$ der Befragten aus stationärer und $44 \%$ aus ambulanter Pflege sehen hier ein großes Potential.

\subsection{Risiken der Zusammenarbeit mit externen Homecare- Dienstleistern}

\subsubsection{Einschätzung der Risiken durch niedergelassene Ärzte}

Befragt man die niedergelassenen Ärzte zu den möglichen Gefahren, die sie in der Zusammenarbeit mit externen Homecare-Dienstleistern vermuten, wird keines der abgefragten Risiken mehrheitlich als groß wahrgenommen (vgl. Abbildung 36). Allerdings sieht die Mehrheit jeweils kleinere Risiken, die wenigsten vermuten gar keine Risiken in Bezug auf die einzelnen Aspekte. Die meisten Ärzte (79\%) befürchten in ihrer ärztlichen Weisungsbefugnis eingeschränkt zu werden - darunter schätzten $38 \%$ das Risiko als groß 


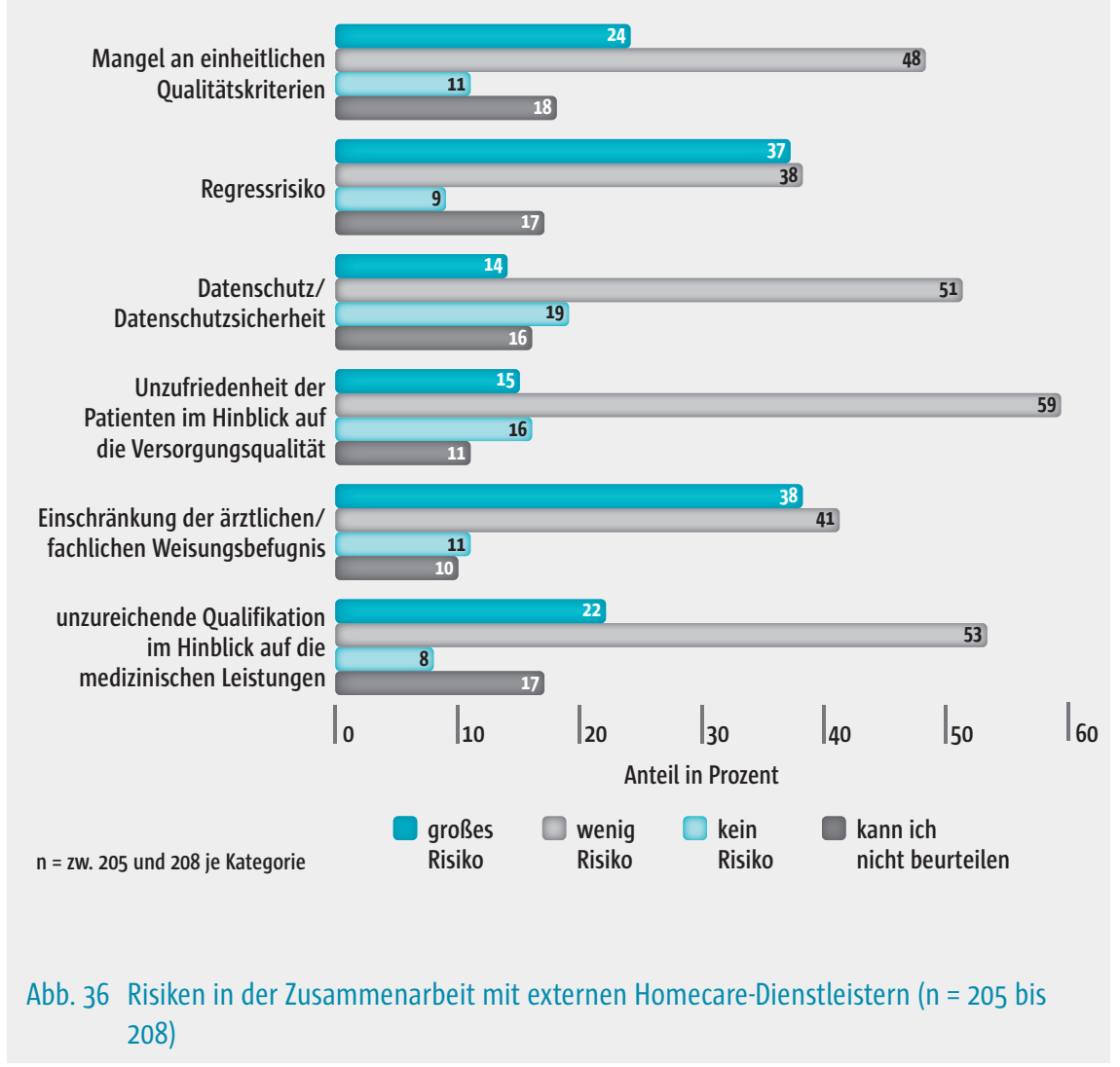

ein. Ebenso sieht die Mehrheit der Ärzte eine Gefahr in der unzureichenden Qualifikation der Homecare-Mitarbeiter im Hinblick auf medizinische Leistungen, der Unzufriedenheit der Patienten mit der Versorgungsqualität (74$75 \%$ ) sowie in dem wirtschaftlichen Risiko für die Praxis (Regressrisiko) (74\%). Interessant ist, dass der Aspekt des Datenschutzes und der Datensicherheit von am wenigsten als ein Risiko eingeschätzt wird. Auffällig ist bei dieser Frage jedoch auch der insgesamt relativ hohe Teil an „kann ich nicht beurteilen“-Antworten. Möglicherweise zeigt sich hier, dass Ärzte zu wenig über die möglichen Risiken, ihre Rechte und ihre Pflichten in der Zusammenarbeit mit externen Homecare-Unternehmen informiert sind und nicht wissen, wie sie sich effektiv vor den genannten Risiken absichern können.

Tabelle 12 zeigt die signifikanten durchgehend negativ Zusammenhänge zwischen der Bewertung der Risiken und der Inanspruchnahme von Homecare-Dienstleistungen durch die Ärzte. Auffällig ist, dass insbesondere Ärzte, die eine Einschränkung ihrer Weisungsbefugnis als Gefahr sehen, weniger Homecare-Dienstleistungen in Anspruch nehmen. 
Tab. 12 Zusammenhang zwischen den Risiken der Zusammenarbeit und der Inanspruchnahme von Homecare-Leistungen ( $\mathrm{n}=118$ bis 138)

\begin{tabular}{|c|c|c|c|c|c|c|}
\hline \multirow{3}{*}{$\begin{array}{l}\text { Risiken der } \\
\text { Zusammen- } \\
\text { arbeit }\end{array}$} & \multicolumn{6}{|c|}{ In Anspruch genommene Homecare-Dienstleistung } \\
\hline & $\begin{array}{c}\text { Diagnosen/ } \\
\text { Befunde }\end{array}$ & $\begin{array}{l}\text { Therapie- } \\
\text { vorschlag }\end{array}$ & $\begin{array}{l}\text { Wirtschaft- } \\
\text { lichkeitsga- } \\
\text { rantie }\end{array}$ & $\begin{array}{l}\text { Empfang } \\
\text { von Rezep- } \\
\text { ten }\end{array}$ & $\begin{array}{c}\text { Therapiean- } \\
\text { passung }\end{array}$ & $\begin{array}{l}\text { Kommuni- } \\
\text { kation }\end{array}$ \\
\hline & $\begin{array}{l}\text { Gamma } \\
\text { (p-Wert) }\end{array}$ & $\begin{array}{l}\text { Gamma } \\
\text { (p-Wert) }\end{array}$ & $\begin{array}{l}\text { Gamma } \\
\text { (p-Wert) }\end{array}$ & $\begin{array}{l}\text { Gamma } \\
\text { (p-Wert) }\end{array}$ & $\begin{array}{l}\text { Gamma } \\
\text { (p-Wert) }\end{array}$ & $\begin{array}{l}\text { Gamma } \\
\text { (p-Wert) }\end{array}$ \\
\hline $\begin{array}{l}\text { Unzureichende } \\
\text { Qualifikation }\end{array}$ & $\begin{array}{l}-0,359 \\
(0,019)^{*}\end{array}$ & - & - & - & - & - \\
\hline $\begin{array}{l}\text { Einschränkung } \\
\text { der Weisungs- } \\
\text { befugnis }\end{array}$ & $\begin{array}{l}-0,333 \\
(0,011)^{*}\end{array}$ & $\begin{array}{l}-0,247 \\
(0,043)^{*}\end{array}$ & $\begin{array}{l}-0,397 \\
(0,010)^{*}\end{array}$ & $\begin{array}{l}-0,327 \\
(0,018)^{*}\end{array}$ & $\begin{array}{l}-0,480 \\
(0,000)^{* * *}\end{array}$ & $\begin{array}{l}-0,476 \\
(0,000)^{* * *}\end{array}$ \\
\hline Regressrisiko & - & - & $\begin{array}{l}-0,542 \\
(0,001)^{* * *}\end{array}$ & - & $\begin{array}{l}-0,308 \\
(0,022)^{*}\end{array}$ & $\begin{array}{l}-0,295 \\
(0,018)^{*}\end{array}$ \\
\hline $\begin{array}{l}\text { Mangel an } \\
\text { Qualitäts- } \\
\text { kriterien }\end{array}$ & $\begin{array}{l}-0,372 \\
(0,012)^{*}\end{array}$ & - & $\begin{array}{l}-0,493 \\
(0,002)^{* *}\end{array}$ & $\begin{array}{l}-0,304 \\
(0,038)^{*}\end{array}$ & - & $\begin{array}{l}-0,311 \\
(0,038)^{*}\end{array}$ \\
\hline$* * *$ p-Wert & ** & & & & & \\
\hline
\end{tabular}

Ebenso sind Ärzte, die große Risiken in der Homecare-Zusammenarbeit befürchten, auch diejenigen die eher von negativen Erfahrungen mit ihrem Homecare-Dienstleister berichten. Insbesondere die Gefahren der Einschränkung der Weisungsbefugnis, unzufriedener Patienten sowie mangelnder Qualitätskriterien werden nach schlechten oder auch nur nicht vorhandenen Erfahrungen mit dem Dienstleistern höher eingeschätzt (vgl. Tabelle 13).

\subsubsection{Einschätzung der Risiken durch Vertreter anderer Berufsgruppen}

Risiken, die von den Klinikmitarbeitern in der Zusammenarbeit mit externen Homecare-Dienstleistern gesehen werden, sind in Abbildung 37 dargestellt. Insgesamt werden die Risiken mehrheitlich als gering oder nicht vorhanden eingestuft. Am häufigsten wird ein Risiko im Mangel an einheitlichen Prozessabläufen gesehen (41\% großes Risiko, 34\% geringes Risiko). Eine unzureichende Qualifikation im Hinblick auf die medizinischen Leistungen wird von $36 \%$ als großes Risiko eingeschätzt, $49 \%$ sehen hierin ein geringes oder kein Risiko. Ein ungesicherter Haftungsübergang von der Klinik zum Arzt sowie die Unzufriedenheit der Patienten bzgl. der Versorgungsqualität werden von den wenigsten Klinikmitarbeitern als Risiken angesehen. 37\% bzw. $10 \%$ sehen hier nur geringe bzw. keine Risiken. 
5 Schriftliche Befragung von Arztpraxen, Kliniken, Pflegeheimen und Pflegediensten

Tab. 13 Zusammenhang zwischen den erwarteten Risiken und der Bewertung der Zusammenarbeit von Homecare-Dienstleistern ( $n=149$ bis 163)

\begin{tabular}{lcc}
\hline Risiken der Zusammenarbeit & $\begin{array}{c}\text { 1. Homecare-Dienstleister } \\
\text { Gamma } \\
(\mathrm{p} \text {-Wert) }\end{array}$ & $\begin{array}{c}\text { 2. Homecare-Dienstleister**** } \\
\text { Gamma } \\
\text { (p-Wert) }\end{array}$ \\
\hline Unzureichende Qualifikation & $-0,201(0,096)$ & $-0,333(0,025)^{*}$ \\
\hline $\begin{array}{l}\text { Einschränkung der Weisungs- } \\
\text { befugnis }\end{array}$ & $-0,389(0,000)^{* * *}$ & $-0,483(0,000)^{* * *}$ \\
\hline Unzufriedenheit der Patienten & $-0,452(0,000)^{* * *}$ & $-0,161(0,321)$ \\
\hline Datenschutzsicherheit & $-0,280(0,019)^{*}$ & $-0,440(0,004)^{* *}$ \\
\hline Regressrisiko & $-0,352(0,002)^{* *}$ & $-0,217(0,132)$ \\
\hline Mangel an Qualitätskriterien & $-0,343(0,002)^{* *}$ & $-0,418(0,001)^{* * *}$ \\
\hline$* * * * n=91$ bis 100, ${ }^{* * *}$ p-Wert $\leq 0,001,{ }^{* *}$ p-Wert $\leq 0,005,{ }^{*} \mathrm{p}$-Wert $\leq 0,05$ & \\
\hline
\end{tabular}

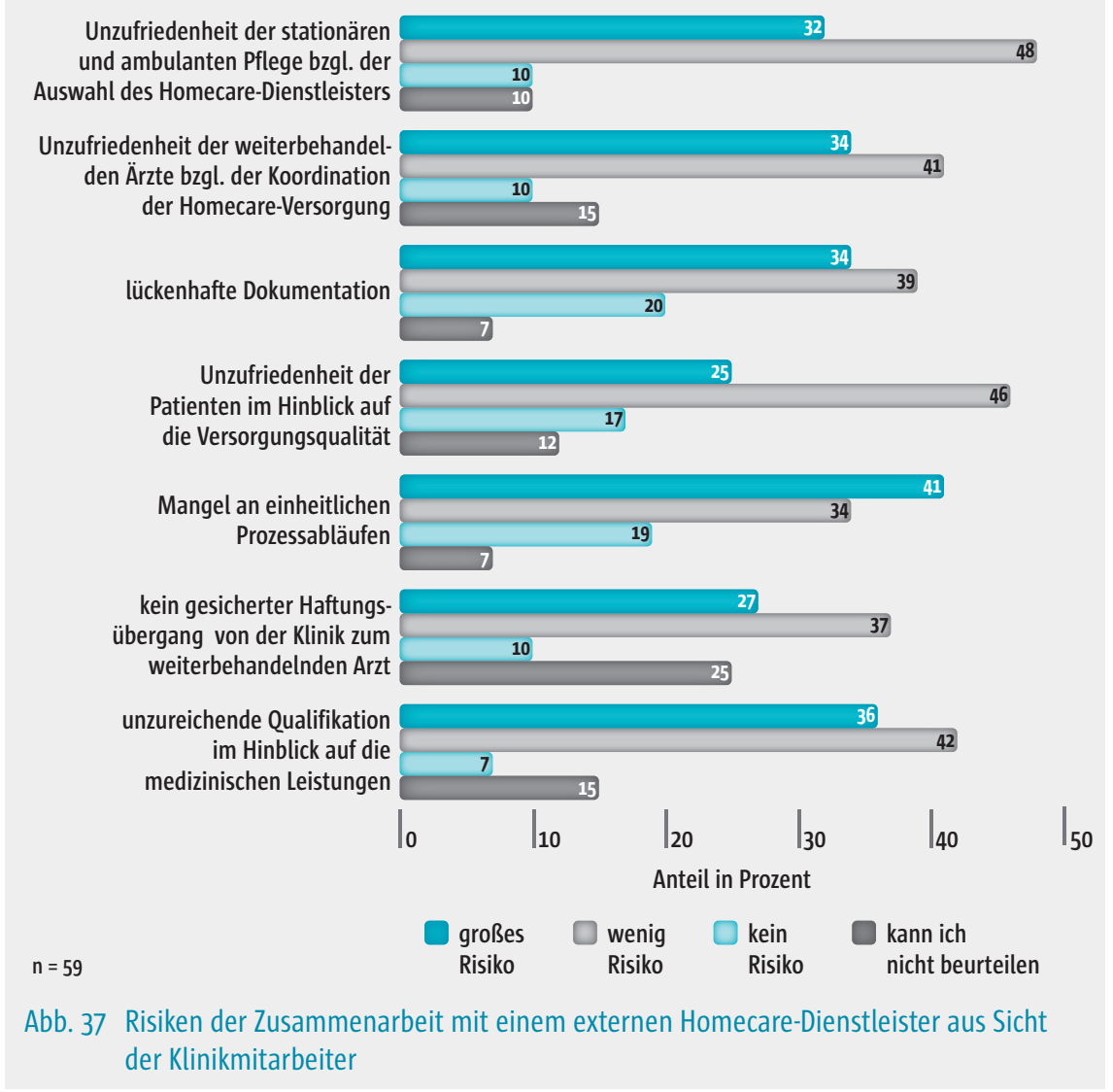


Negative Zusammenhänge lassen sich auch hier zwischen den wahrgenommenen Risiken und der Bewertung der Zusammenarbeit mit dem HomecareDienstleister nachweisen. Wie Tabelle 14 zeigt, haben Klinikmitarbeiter, die große Risiken in der Zusammenarbeit mit Homecare-Dienstleistern in den aufgelisteten Aspekten sehen, eher negative Erfahrungen mit ihrem Homecare-Dienstleister gesammelt als jene, die diese Risiken geringer bzw. als nicht vorhanden einschätzen.

Insgesamt noch weniger Risiken sehen Vertreter aus stationärer Pflege in der Zusammenarbeit mit externen Homecare-Dienstleistern (vgl. Abbildung 38). Gefahren werden am ehesten in dem Mangel an einheitlichen Qualitätskriterien (22\%), unzureichende Qualifikation im Hinblick auf die medizinischen Leistungen $(17 \%)$ sowie einer lückenhaften Dokumentation (16\%) gesehen. Der Anteil, der diese Aspekte als geringe oder keine Risiken einschätzt, liegt jedoch mit 74\%, 77\% und 79\% der Befragten deutlich höher. Aspekte des Datenschutzes spielen bei der Einschätzung der Risiken die geringste Rolle.

Sehr ähnliche Größenordnungen lassen sich für den ambulanten Pflegebereich zeigen.

\subsection{Zukunft der Homecare-Versorgung}

Etwa die Hälfte der Ärzte denkt nicht, dass eine VerAH die Dienstleistungen eines externen Homecare-Unternehmens vollständig ersetzen kann und hält daher auch die Einstellung von speziell qualifiziertem Personal (z.B. eine VerAH) in ihrer eigenen Praxis für keine geeignete Maßnahme zur Verbesserung der Versorgung ihrer Homecare-Patienten (vgl. Abbildung 39). 79\% der Ärzte können sich ganz oder teilweise vorstellen, mit einer praxisexternen

Tab. 14 Zusammenhang zwischen den Risiken der Zusammenarbeit und der Bewertung der Zusammenarbeit mit Homecare-Dienstleistern ( $n=38$ bis 48 )

\begin{tabular}{l|l}
\hline Risiken der Zusammenarbeit & $\begin{array}{c}\text { 1. Homecare-Dienstleister } \\
\text { Gamma } \\
\text { (p-Wert) }\end{array}$ \\
$\begin{array}{l}\text { Unzureichende Qualifikation im Hinblick auf die medizinischen } \\
\text { Leistungen }\end{array}$ & $-0,444(0,040)^{*}$ \\
\hline $\begin{array}{l}\text { Kein gesicherter Haftungsübergang von der Klinik zum weiterbe- } \\
\text { handelnden Arzt }\end{array}$ & $-0,463(0,018)^{*}$ \\
\hline $\begin{array}{l}\text { Unzufriedenheit der Patienten im Hinblick auf die Versorgungs- } \\
\text { qualität }\end{array}$ & $-0,520(0,012)^{*}$ \\
\hline \begin{tabular}{l} 
Lückenhafte Dokumentation \\
\hline$* * *$
\end{tabular} -Wert $\leq 0,001,{ }^{* *}$ p-Wert $\leq 0,005,{ }^{*}$ p-Wert $\leq 0,05$ & $-0,444(0,018)^{*}$ \\
\hline
\end{tabular}



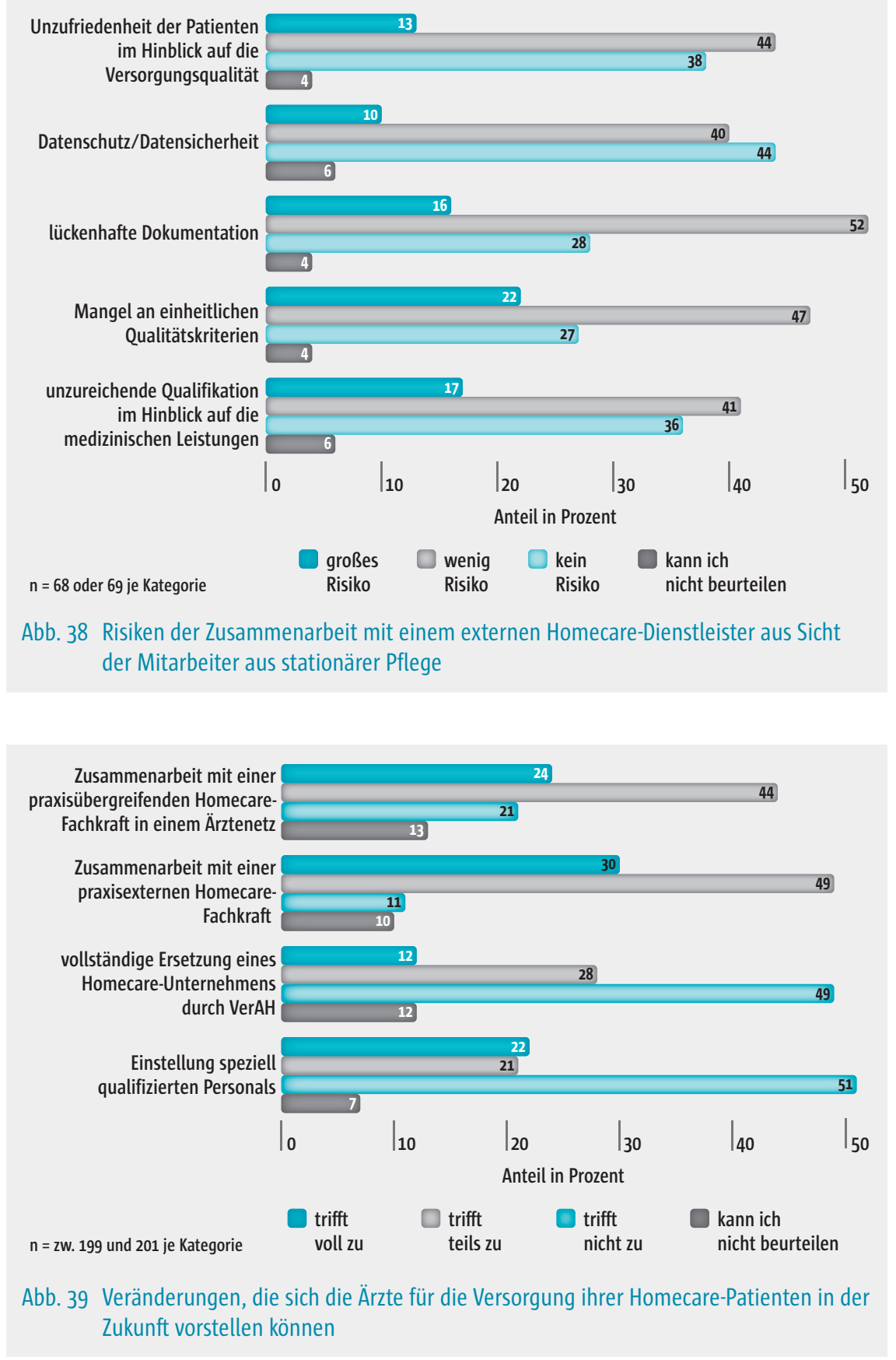


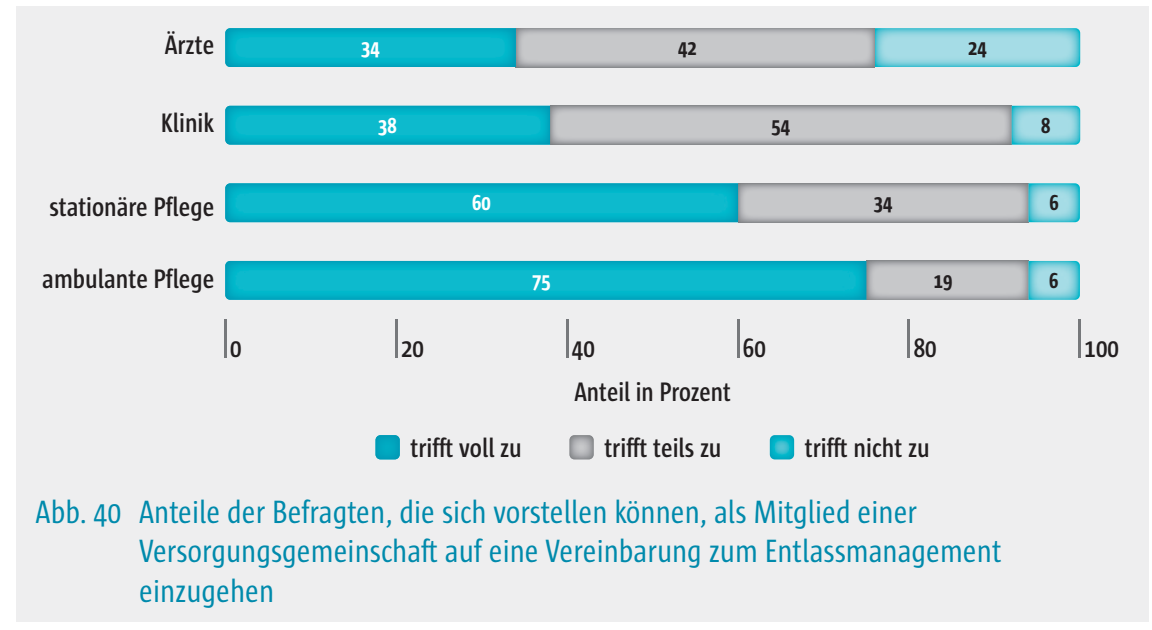

Homecare-Fachkraft zusammenzuarbeiten, die die notwendigen Qualifikationen und Kompetenzen besitzt. Für 67\% der Ärzte ist es eine Option, mit einer qualifizierten Homecare-Fachkraft zusammenzuarbeiten, die praxisübergreifend in einem Ärztenetz eingebunden ist.

67\% der Ärzte können sich vorstellen, als Mitglied einer Versorgungsgemeinschaft eine Vereinbarung über das Entlassmanagement mit einer Klinik abzuschließen (vgl. Abbildung 40). In der stationären Pflege sind 57\% bereit dazu, in der ambulanten Pflege sogar $72 \%$. Im Gegensatz dazu wären nur $35 \%$ der Befragten aus Kliniken dazu bereit, wobei es für knapp die Hälfte der Befragten zumindest teilweise eine Option darstellt.

Ebenfalls 67\% der Ärzte können sich vorstellen, dass Krankenkassen künftig eine gesonderte Vergütung der Homecare-Dienstleistung vorsehen werden. Im Klinikbereich glauben 32\% daran und 24\% teilweise. In der stationären und ambulanten Pflege glauben jeweils 35\% bzw. 40\% der Befragten an diese Option. 